ALPHA No 26 / Julio 2008 (153-166)

ISSN 0716-4254

http://alpha.ulagos.cl

\title{
LA APROPIACIÓN DE(L) SENTIDO: LAS EXPERIENCIAS HERMENÉUTICAS DE DIÁLOGO Y COMPRENSIÓN A PARTIR DE GADAMER
}

The Appropriation of Meaning: the hermeneutic experiences of dialogue and comprehension

Fernando J. Vergara Henríquez*

Resumen

El siguiente ensayo intenta clarificar la experiencia hermenéutica a partir del concepto de "comprensión", entendiéndolo como la puesta de acuerdo con el otro sobre algo a través del lenguaje como medio universal cuyo fin es el consenso. De allí que el "diálogo" sea la concreción de la comprensión en aras de la correcta interpretación, acción encerrada en la dialéctica de la "pregunta” y la "respuesta" en un círculo comprensor y, justamente, esta dimensión lingüística de la comprensión señala el acopio de la conciencia de la historia efectual, donde la tradición consiste en el medio del lenguaje situado en un horizonte en función de las interpretaciones y en fusión de perspectivas.

Palabras claves: Hermenéutica, comprensión, diálogo, historia efectual.

The following essay seeks to clarify hermeneutic experience in terms of "understanding", conceived of as arriving at an agreement with another on something through language as a universal medium whose purpose is consensus. On this basis "dialogue" is the concretion of understanding that seeks correct interpretation, enclosed within the dialectic of "question" and "answer" that form a circle of understanding, in which the linguistic dimension of understanding is indicative of the accumulative effects of history upon consciousness, and where tradition is defined through the medium of language that is located in a horizon whose function is the interpretation and the fusion of perspectives.

Key words: Hermeneutic, understanding, dialogue, effectual history.

En tanto la teoría filosófica, la hermenéutica, a pesar de ser considerada a principios del siglo XX como una disciplina menor en el campo de los estudios humanísticos — como arte o técnica para la guía de la interpretación de textos de la tradición cultural con el fin de zanjar la distancia temporal, espacial y cultural e incorporar el sentido a la comprensión actual— pasó a 


\section{Fernando J. Vergara}

convertirse en una amplia concepción y perspectiva filosófico-cultural hacia nuevas formas de pensar. Vattimo ha hecho famosa la expresión de que la hermenéutica constituye una nueva koiné como "evento plurilingüístico de destino", paradigma compartido y discurso filosófico actual. Es decir, el nuevo lenguaje ecuménico de la cultura contemporánea que versa sobre la esencia del sentido o sobre la tendencia profunda de nuestra época que abre el horizonte histórico-cultural de sentido al reconocerle "una centralidad, que se testimonia por la presencia misma del término, de las temáticas hermenéuticas y de los textos a que las impone, en los debates, en la enseñanza, en los cursos universitarios, y hasta en aquellos terrenos como la medicina, la sociología o la arquitectura, que buscan establecer con la filosofía un nuevo vínculo (1991:56). En otro lugar, Vattimo se refiere a la hermenéutica filosófica gadameriana como "una verdadera y propia “ontología de la actualidad”, una filosofía del mundo tardomoderno donde el mundo se disuelve efectivamente, y cada vez más, de modo global, en el juego de las interpretaciones (68). Grondin, por su parte, afirma que "la hermenéutica es un problema universal. Lo que le otorga [...] el estatuto de prima philosophia de nuestro tiempo [debido a la] omnipresencia del fenómeno interpretativo” (2003:35). Desde otra perspectiva, Rorty se refiere a la "hermenéutica" no como "una disciplina, ni [...] un método de conseguir los resultados que la epistemología no consiguió obtener, ni [...] un programa de investigación. Por el contrario, la hermenéutica es una expresión de esperanza de que el espacio cultural dejado por el abandono de la epistemología no llegue a llenarse, que nuestra cultura sea una cultura en la que ya no se siente la exigencia de constricción y confrontación” (287, 288); confrontación que se realiza respecto a los objetos del pensamiento que limitan la investigación como deseo de fundamento de todo discurso. Y el propio Gadamer, pensando la "hermenéutica" en tanto interpretación, destaca que este término haya tenido "como pocos la rara fortuna de expresar de forma simbólica la actitud de toda nuestra época" (1993:43). Gadamer y su "neohermenéutica” u "ontología existencial” como "filosofía de la comprensión”, encuentra la articulación de perspectiva y de metodología filosófico-hermeneuta cuyo problema central -a saber, la concepción del lenguaje y de la razón como interpretación-comprensión de ese lenguaje o ser del lenguaje- es considerado en forma omniabarcante y universal, pues la hermenéutica gadameriana emprenderá una meditación fenomenológica sobre el enraizamiento esencial de la comprensión como aquella condición histórica y existencial del sujeto. 
La apropiación de(l) sentido: Las experiencias hermenéuticas

\section{GADAMER Y LA FILOSOFÍA HERMENÉUTICA DEL SENTIDO}

El giro hacia el lenguaje realizado en el siglo $\mathrm{XX}$ por la filosofía se relaciona con el "derrumbamiento" de la metafísica como pensamiento fundamentador de la cultura. El sujeto y la conciencia operan expuestos a la fragmentación nihilista de una realidad que no transa sus transformaciones materiales. Así, la filosofía renuncia a la reflexión sobre conciencia y realidad, apostando por una meditación sobre el hombre como ser-en-elmundo y por el mundo como nuestro mundo, quedando coimplicados como ser-en-el-lenguaje. Este giro o torsión a la habitual manera de entender la acción humana con su palabra — lenguaje — y a la acostumbrada forma de recepción del flujo histórico — sentido - " "apunta, según Gadamer, a una estructura universal-ontológica, a la constitución fundamental de todo aquello hacia lo que puede volverse la comprensión. El ser que puede ser comprendido es lenguaje” (2005:567).

Un "giro ontológico" realiza Heidegger a los conceptos de comprensión e interpretación, interpretación ligada a la "facticidad". Estamos arrojados a un mundo que posee un orden y unas relaciones de funcionamiento que, finalmente, le otorga un sentido tal que nuestro acceso al mundo se lleva a cabo como un acceso comprensivo/interpretativo. En consecuencia, la comprensión y la interpretación dependerán de la proyección que hace la existencia de ese sentido-sobre-el-mundo- como horizonte de proyección y de mediación articuladora ("hacia donde”, “desde el cual” algo resulta comprensible "como algo", 1998:172-177). Lo expuesto impulsa el "giro hermenéutico" gadameriano en la filosofía, que hace fecunda las intuiciones de su maestro respecto el proceso de comprensión y de interpretación desarrollado por las ciencias del espíritu, ampliando el campo de comprensión a partir de la categoría dialógica como un saber peculiar que se hace cargo de "lo aún no dicho", considerando ese "todavía" una suerte de fuente de significación por explorar y explotar, pues ambos definieron hermenéutica como la autocomprensión, la cual no sería otra cosa sino la comprensión del propio ser como ser-en-el-mundo. Para Gadamer, entonces, la comprensión es la interpretación lingüística de los fenómenos que experimentamos en la vida, entendida como fuente de sentido. Descubre Gadamer que la hermenéutica es una experiencia más amplia que la conciencia del sujeto. Es decir, la experiencia de ser en el tiempo, de que el tiempo es el ser y, como tal, es la manera en que la vida humana revela al ser que le comprende, pues somos seres de sentido "arrojados" a un mundo en el que coparticipamos en su conformación y transformación. La conciencia así entendiendo, es conciencia en el aparecer de(l) sentido. Este ser como tiempo, que es la comprensión, es la condición ontológica de la existencia humana, pues antes que la conciencia 


\section{Fernando J. Vergara}

tenga contenido $\longrightarrow$ antes de autocom-prendernos - ya estamos en la comprensión por situarnos al interior de la historia. El problema de la interpretación, entonces, aparece ligado a la olvidada pregunta por el ser como "aquello sobre lo cual nos interrogamos es la pregunta por el sentido del ser" (Ricoeur, 2001:83), pregunta que expresa el compromiso que debe asumir la conciencia en su intento por reintegrarse al circuito histórico.

La hermenéutica, aquí, nombra el "carácter fundamentalmente móvil del estar ahí, que constituye su finitud y su especificidad y que, por lo tanto, abarca el conjunto de su experiencia en el mundo. Que el movimiento de la comprensión sea abarcante y universal no es arbitrariedad ni inflación constructiva de un aspecto universal, sino que está en la naturaleza misma de la cosa” (2005:12). El Dasein es el lugar, el topos desde donde surge la pregunta por el ser o la epifanía comprensiva de su ser. El Dasein se encuentra siempre en situación y proyectado en su comprender, despliegue al que Heidegger llama interpretación. Mediante esta condición el Dasein proyecta su ser como poder-ser; como pura apertura a la posibilidad de ser, vale decir, como "sentido" que hace posible que algo sea comprensible en un horizonte concebido para la existencia. El carácter específico de la comprensión viene dado por la finitud de la experiencia humana como totalidad, y la movilidad de la experiencia humana de finitud está determinada por las formas siempre provisionales de la comprensión.

La comprensión indica un proceso que tiene como centro al lenguaje, en cuanto forjador de realidades y acervo de perspectivas - hombre (ser/espíritu/razón) y realidad (ser/naturaleza/mundo)— - y, como tal, posee un carácter ontológico como realidad esencial, pues posibilita la posesión de un mundo y hace posible todo tipo de interpretación en el espacio de la historia y de la tradición. La propuesta de Gadamer por una "universalidad del lenguaje” radica en que envuelve a la experiencia lingüística del apalabrar sobre el mundo como medio de exhibición manifestativa de(l) sentido en un diálogo entre singularidad y totalidad (2005: 536).

El lenguaje es aquella "realidad intermedia" y "aparece [...] como la verdadera dimensión de la realidad” (Gadamer, 2004:327) que posibilita que exista el mundo y de que se manifieste al hombre como mundo o totalidad ordenada de significaciones en interacción dialéctica. El lenguaje y la tradición histórica articulan la experiencia humana de comprensión presentada bajo la forma de diálogo entre intérprete y texto; entre tiempo e historia en una fusión de horizontes de sentidos —-mediación, integración - como red de inteligibilidad para la comprensión, haciendo posible una real y efectiva transformación en la vida del intérprete debido a la interpretación realizada bajo el signo de la "verdad” (Vattimo, 1996:117). De tal forma, el lenguaje no

es un ente preexistente de manera independiente, sino que anuncia "un todo 
La apropiación de(l) sentido: Las experiencias hermenéuticas

de sentido" y reclama la aparición del mundo de sentido como "acontecer hermenéutico" que le subyace y se expresa en la tradición histórica como eje de subjetividades interpretadoras.

\section{APROPIACIÓN DE(L) SENTIDO: EXPERIENCIAS HERMENÉUTICAS DE DIÁLOGO Y COMPRENSIÓN}

Gadamer presenta en una triple restitución la experiencia hermenéutica: la estética —el juego-, la historia — tradición- y el lenguaje —ser/comprensión - entendiendo por "experiencia hermenéutica” el correlato de la comprensión, es decir, aquella experiencia de apropiación de(l) sentido del comprender.

La experiencia hermenéutica resulta ser intrínsecamente histórica y la comprensión su resultado, por ello, es extrínsecamente metahistórica, debido a que la experiencia y la comprensión se revelan lingüísticamente como expresión y comprensión de un mundo que viene a permanecer delante de nosotros y en nosotros mediados por el lenguaje. Y, a través de este "medio relacionador", la experiencia hermenéutica es dialéctica en el sentido de ampliación e iluminación de la autocomprensión desde el encuentro, el legado que no es precisamente mera captación conceptual, sino un acontecimiento en el que un mundo se le abre como algo que no existía antes. En este acontecimiento, basado en lo lingüístico y posibilitado por la dialéctica con el significado transmitido, la experiencia hermenéutica encuentra y alcanza su realización. Asimismo, “comprensión” y "lenguaje” cumplen una función ontológica, dando el carácter de ontológica a la experiencia hermenéutica en tanto que revelan el ser de las cosas iluminándolo y revelando "lo que significa ser” más allá del simple ser de un objeto o cosa. Por ello, la hermenéutica es una experiencia del "acontecer del lenguaje" que hace el mundo comprensible para nosotros. Y este acontecimiento no habla de otra cosa sino de la manifestación veritativa del ser en tanto que des-ocultamiento, más bien, un ocultamiento simultáneo de la verdad en su plenitud inagotable. La verdad no es un hecho, "ocurre" al igual que la hermenéutica y su "acontecer experiencial".

En la abierta región hermenéutica, partir de "aquello que es" no significa otra cosa sino retornar a la pregunta original sobre la comprensión y dirigirse fenomenológicamente "a las cosas mismas". Gadamer se sirve de algunas ideas de la fuente fenomenológica husserliana. De ellas, tres le son fundamentales: primero, la importancia concedida al problema del "sentido" y su horizonte constitutivo de lenguaje como función reveladora del mundo; segundo, la noción de "horizonte" como encuadre en el cual se muestra y se constituye el sentido; tercero, el concepto de "mundo de la vida” o ámbito de 


\section{Fernando J. Vergara}

experiencia previo a la actividad noética o reflexiva como receptáculo desde y en el cual la vida ocurre históricamente y discurre lingüísticamente, previamente a toda objetivación científica como a toda reflexión filosófica y supone una suerte de red en la que remite todo sentido constituido y desde el que se reactiva toda metodología objetivante tanto de la ciencia como de la filosofía.

Preguntar por el "ser de la comprensión” o “en qué modo comprender es ser” estará bajo la forma lingüística — eje de la experiencia de apropiación de(l) sentido de la comprensión- de diálogo histórico: "el ser que puede ser entendido está lingüísticamente articulado, es lenguaje” (2005: 23) —que expresa la relación entre lingüisticidad y comprensibilidad del ser- y esa articulación es el diálogo y ese entender que nos tiene prendidos, es la comprensión. En esto, propone Gadamer la descripción de "lo que hay” en la tradición y en la común participación del solidario sentido comunitario con el que se orienta al sentido y por el que nos comprendemos sin recurrir a la necesidad de reducirlos a objetos científicos. La clave en esta proposición es la hermenéutica: un "camino" hacia la comprensión, una búsqueda interpretativa de la comprensión mediada por el lenguaje inscrito en la historia.

Todo lo que el hombre puede experimentar se incluye en esta esfera de la experiencia de mundo de manera abarcante sobre el fenómeno de la comprensión: un contenido de verdad que penetra y actúa en el ámbito de nuestra existencia. La comprensión no es un modo o aspecto meramente gnoseológico, sino un constitutivo ontológico del ser del hombre, pues rebasa la radical finitud del comprender, toda fundamentación última como, asimismo, toda pretensión de irrefutabilidad absoluta, siendo el comprender pura posibilidad de ser como rasgo ontológico de un ser que es puro proyecto de ser en lo "histórico" (2005: 327).

Además, toda comprensión es mediata, pues se encuentra en medio de conformaciones históricas, prejuicios, preopiniones; valoraciones que delinean toda comprensión que, a su vez, es la estructura previa de toda interpretación como despliegue de esta misma estructura comprensiva (2005:325). Por ello, se hace "visible la estructura de la comprensión histórica en toda su fundamentación ontológica, sobre la base de la futuridad existencial del estar ahí humano" (2005:326). Comprender supone estar siempre pendiente del hacer de la historia y de la tradición que determinan al sujeto en el aquí y el ahora y que provoca la apertura hacia el diálogo que es la mecánica de la comprensión (2005:360).

La pretensión de universalidad es fundamental para entender el proyecto hermenéutico de Gadamer. El "aspecto universal de la hermenéutica” radica en el lenguaje y en el carácter lingüístico de entender 
La apropiación de(l) sentido: Las experiencias hermenéuticas

(2005: 451) planteando una "pregunta universal” (2005: 458) al mundo y reconociendo la propia finitud del comprender que alcanza infinitud en la historia como estructura ontológica del ser del hombre como ser histórico.

\section{DIÁLOGO}

Para la hermenéutica filosófica, además de la función primaria del lenguaje, es decir, aquella función nominativa o designativa —recabar y ordenar información sobre hechos que se dan en el mundo-, el lenguaje cumple un uso primordial: hacer posible un mundo plurisignificativo; comprenderlo y verbalizar tal comprensión mostrando el sentido y las significaciones que se producen desde el diálogo comprensivo entre seres humanos, según afirma Domingo Moratalla, como una

"comunicación mantenida o acontecimiento relacional que tiene por objeto la comprensión de aquello sobre lo que se conversa y de aquél con quien se conversa. Y si el diálogo es camino de conocimiento de la realidad y del otro hombre, es también el método de "realización” y "socialización", ya que somos lo que somos gracias a nuestra relación con los otros y con la realidad que con ellos habitamos. Así, el mundo se nos presenta no sólo como lugar en el que estamos, sino como obra que realizamos y compartimos [como fruto de] la rectitud de la opinión y llegar a la verdad pasa por el esfuerzo de la definición, el esfuerzo en la búsqueda compartida de la palabra, de la fijación de un sentido donde uno y otro, uno con otro, buscamos instalarnos en lo común que llamamos universales (1991: 177-178).

Para Gadamer, la comprensión - en tanto que reconstrucción de una estructura de sentido - entraña el primado del diálogo, es decir, "salir de sí mismo, pensar con el otro y volver sobre sí mismo como otro" (2004:356). Más que un instrumento, el lenguaje es un "órgano" mediador entre el sujeto y objeto instaurando un "mundo intermedio" y, como tal, asienta sentido, pues "la interpretación ya no es considerada como un "modo de conocer" sino como el "modo de ser" constitutivo del ser humano y va a quedar vinculado a la palabra, [...] en tanto que auténtico medio de su realización efectiva en el interior del diálogo, de la comunicación, de la convivencia” (Garagalza, 2002: 47-48) histórica en el horizonte de la comprensión.

El diálogo o experiencia comprensiva de fusión de perspectivas es en donde se presenta la primacía hermenéutica de la pregunta como experiencia de íntima necesidad por superar la limitación de nuestros respectivos horizontes. Resulta esencial a toda pregunta que tenga el sentido de una orientación, pues con la pregunta, lo preguntado es colocado bajo una 


\section{Fernando J. Vergara}

determinada perspectiva hacia el encuentro con la respuesta, en una dialéctica de intercambio entre un sujeto que "escucha" y un objeto que se revela. Que surja una pregunta supone siempre introducir una cierta ruptura en el ser de lo preguntado. El logos que desarrolla este ser quebrantado es, en esta medida, siempre ya respuesta y sólo tiene sentido en el sentido de la pregunta. La apertura de la pregunta está en la base de la estructura de la experiencia hermenéutica: la latencia de una respuesta implica, a su vez, que aquél que pregunta es alcanzado e interpelado por la misma corriente de la tradición en que habita ese texto. El diálogo funde los horizontes de(l) lenguaje como estructura comprensora del "círculo hermenéutico" con el mundo.

El modelo dialógico — como se expone aquí- manifiesta la apertura dada por la alteridad, una alteridad rebasada por el diálogo como experiencia de apropiación comprensiva y como horizonte de sentido bajo el régimen de expresión de su potencial crítico-reflexivo como aplicación (2005: 380) de una racionalidad dialógico-experiencial (2005: 333).

El "diálogo que somos” ocurre en la copertenencia o, más bien, en la coimpertinencia entre dos sujetos sobre "aquello que" acontece lo que hace surgir una suerte de experiencia humana de mundo cuando se interpreta y se comprende, lo que co(i)mplica a ambos sujetos en la fusión dialógica del lenguaje, en el habla, en la conversación (2004:242) ahora involucrados en todo proceso de apropiación comprensiva (2004: 112).

El telos dialógico gadameriano no es otro sino la "comprensión” —-formación, educación, humanismo-, en el sentido del entendimiento entre quienes dialogan genuinamente sobre aquello en que se funda lo que viene dado por una orientación compartida, recíproca hacia y sobre el asunto de la conversación, apareciendo complementarios en el marco de la "comprensión dialógica” (2005:461-478) y en la experiencia vital y subjetiva —limitada, finita y provisional — de la realidad y del mundo.

La apertura realizada por la experiencia de apropiación de(l) sentido de la comprensión no habla de una hermenéutica como "práctica” humana, sino que concibe como su telos al entendimiento desde la comunicación dialógica, que en su despliegue surge como un quehacer práctico que corresponde a un cierto ethos (2004:293-308).

Entonces, el núcleo de la hermenéutica filosófica gadameriana se encuentra en la "experiencia". Más aún, consideramos a esta experiencia como aquélla de la comprensión y como comprensión experiencial de un sujeto bajo determinaciones históricas que funda posibilidades de sentido lingüísticamente expresadas. El significado que se otorga a esta forma de experiencia se entiende como un "acontecer" de mediación recíproca "del pensamiento con la vida actual” (2005:222) y no como dominio del sujeto 
La apropiación de(l) sentido: Las experiencias hermenéuticas

sobre el objeto: una coimplicación entre sujeto y objeto posibilitada por la fusión del comprender y el comprender-se.

El diálogo, en fin, es una actividad que da sentido a la vida humana "desde la finitud de la existencia situada" (1991:196) y que nos "tiene tomados" en nuestra historicidad; nos "incorpora" a una comunidad dialogante o sensus communis que se nutre de lo verosímil, es decir, de la equivalencia entre las palabras en búsqueda de un sentido que es especificado por la tradición histórica desde la finitud.

El quehacer histórico del pensamiento se aloja en la conciencia dialógicamente fundada, pues "es cierto que si un día se acaba el preguntar, se habría acabado también el pensamiento” (2004:224-238). El conocer y el conocer-se son experiencias históricas; constituyen el fundamento comprensor de la historia, y como tal, son una forma de efecto que se sabe a sí misma como "efectual". La "eficacia de la historia" o los "efectos de la historia en nuestra conciencia", refieren al hecho de que cada interpretación de la historia es, a su vez, histórica y viceversa; es decir, que tal acontecimiento histórico no quede recluido en el acontecer, sino que trascienda proyectando su influencia en el futuro, permaneciendo en sus efectos que no son otra cosa, sino las interpretaciones que provoca en el tiempo.

La comprensión — tal como se trata aquí- de los acontecimientos históricos y la acción de autocomprensión no puede hacerse bajo el modelo científico-técnico o conforme a criterios de la lógica administrativoburocrática de la vida social contemporánea, pues no se trata de un modo de conocimiento, sino un modo de ser: el modo de este ser que existe al comprender como, asimismo, la implicación que hace la historia de nuestra conciencia como conciencia histórico-efectual.

El plan de Gadamer es un "proyecto [de] ampliación del modelo occidental de racionalidad [enunciativa-técnico-instrumental y tiene como finalidad] promover una racionalidad lingüística que [integre] la potencia mito-poética de la palabra” (Garagalza, 2002:10) y, por ello, Gadamer concibe la hermenéutica como la pregunta filosófica que concierne a la posibilidad de la comprensión en referencia a lo que acontece en la praxis y no en la empiria subjetivista y/o instrumentalista de la comprensión, es decir, en aquello "que ocurre con nosotros por encima de nuestro querer y hacer" (2005: 10). Gadamer piensa a la hermenéutica como un proceso histórico que se define por la coimplicación de sucesos de verdad y de sentido. En el pensamiento gadameriano, "sentido" designa el "estatuto" o "soporte ontológico" del lenguaje como referente interpretativo de lo que se dice. El sentido sería una suerte de idealidad que nos guía en la comprensión. "Fijar" o "establecer" el sentido de la interpretación y encontrar una respuesta a la 


\section{Fernando J. Vergara}

pregunta sobre cómo sea posible la comprensión donde el objeto — porcomprender, que no está inmediatamente dado ni menos garantizada la correcta transmisión y entendimiento de su sentido, es la pregunta central de la hermenéutica gadameriana.

El proyecto hermenéutico de Gadamer referido a la comprensión consiste en "rastrear y mostrar lo que es común a toda manera de comprender: que la comprensión no es nunca un comportamiento subjetivo respecto a un “objeto" dado, sino que pertenece [...] al ser de lo que se comprende" (2004: 13-14). De tal forma, en Gadamer la hermenéutica cobra la figura, por una parte, de una teoría de la verdad y, por otra, la de un método que expresa la universalización del fenómeno interpretativo desde la historicidad concreta y personal del sujeto, y lo hace con el fin de establecerse como una filosofía interpretativa y comprensiva de la experiencia humana de sentido en la historia lingüísticamente expresada.

Desde el modelo clásico de hermenéutica - el análisis del modo como se puede alcanzar una adecuada o correcta interpretación de un texto determinado - es desde donde se establece una íntima relación entre comprender e interpretar: la hermenéutica es esencialmente una forma de comprensión que “pregunta cómo es posible la comprensión” (2005: 12).

Y, en esta línea, la filosofía hermenéutica propuesta por Gadamer tiene un carácter general que analiza las condiciones de posibilidad de todo proceso de comprensión, es decir, del proceso de "comprender-algo-como-algo". La comprensibilidad de "algo como algo" es una herencia heideggeriana, donde se concibe el fenómeno hermenéutico fundamental: el fenómeno hermenéutico de la comprensión que adquiere su articulación en el despliegue de la interpretación como lo abierto originario sobre la proyección anticipativa del comprender existenciario.

\section{COMPRENSIÓN}

Permanecemos, así, en el terreno de la hermenéutica, en la heredad de lo simbólico y representacional e ingresamos al "taller" de manufactura de las significaciones, a la dimensión semiótica del significado y del sentido, en fin, a la urbanización gadameriana de la provincia heideggeriana de la reflexión sobre la hermenéutica del ser: el ser humano capaz de preguntar y preguntarse sobre sí mismo, justamente, aquello que caracteriza su modo de ser. De esa forma el Dasein, el ser aquí y ahora, existe comprendiendo-se, sabiendo-se, pues la "comprensión del ser es, ella misma, una determinación de ser del Dasein” (1998:35).

El "comprender" — referido a la esfera ontológica — es una capacidad para poder "hacer aparecer” y "valer” la forma de darse el Dasein, facilitando 
La apropiación de(l) sentido: Las experiencias hermenéuticas

su "poder-ser": proyectar y dejar que se realice el ser-en-el-mundo sobre el trasfondo mismo del ser.

De tal forma, la analítica existenciaria del Dasein permite descubrirlo como una situación de "comprender", de "sentido" y de "interpretación", desde la aperturidad como forma esencial del ser del Dasein. Tal aperturidad, “estar-en-el-mundo”, equivale a “comprender” más allá de los límites de una forma específica de conocimiento.

Por tanto, en Heidegger, ser y comprender conforman una unidad originaria y existenciaria que define al Dasein como "proyecto" y "posibilidad", enraizando la interpretación a la historia y a la ontología, interpretación en tanto desarrollo del comprender en cuanto se apropia de lo comprendido.

Y esta apropiación interpretativa hace surgir al "sentido" como “aquello en lo que se apoya la comprensibilidad de algo" (1998:175). La comprensión de este modo de ser es aquello que también levanta los límites del ser del Dasein, pues arraiga en este "ser-en-el-mundo" y de esta forma el comprender arraiga en el mundo que habitamos, pertenecemos, conformamos y configuramos, y desde el cual proyectamos nuestra historicidad y coordinamos la adquisición de sentido. En fin, se es y se existe comprendiéndose y sabiéndose y, por ende, se trata de una autocomprensión que configura a la existencia misma. Por lo tanto, se existe en actitud comprensiva, en actitud hermenéutica, siendo la más clara e indicativa manera de ser el preguntar-se por el mismo ser, por el sí mismo.

La consideración de la "comprensión” como el "modo de ser” del ser humano asume, igualmente, por estructura de la comprensión al "círculo hermenéutico" o “círculo del comprender”, lo que resulta central en el pensamiento gadameriano.

Una circularidad comprensora que hace referencia a una llamada “metodología gadameriana de la comprensión”, y que surge en el momento de requerir la comprensión de un texto, se muestra necesaria para anticipar el sentido de su totalidad, a partir de sus partes como piezas que "encajan” en el modelo de sentido anticipado. Comprender la totalidad desde las partes es la condición de posibilidad para la comprensión del contexto global, que a su vez es resultado de la totalidad de partes textuales individuales.

Un análisis fenomenológico de la conciencia históricamente determinada permite a Gadamer definir con claridad los límites de dicha conciencia frente a las pretensiones del subjetivismo, a la estrechez del historicismo con su "objetividad histórica" y al círculo trazado por la hermenéutica romántica, pues "la comprensión no es nunca un comportamiento sólo reproductivo, sino que es a su vez siempre productivo [y como tal] cuando se comprende, se comprende de un modo diferente" (2004: 


\section{Fernando J. Vergara}

366-367). El hecho es que en la comprensión siempre nos encontramos, ya en una situación determinada lo que, por tanto, representa una posición que limita las posibilidades de ver. Pero tales posibilidades de poder ver quedan supeditadas a un punto de vista, ya que no nos es dado un punto absoluto desde el que pudiéramos contemplar todo de manera absoluta. Y ese ámbito de visión que encierra todo lo que es visible desde ese punto de vista determinado es lo que Gadamer llama "horizonte". La tradición aparece como el horizonte de la subjetividad y como posibilidad para la comprensión del sujeto de sí mismo. Tomar conciencia del hecho de que la historia o la tradición actúan en nosotros, significa aceptar la alteridad de la acción recíproca entre “efecto” y "saber” como, también, a la mediación entre "historia" y "verdad".

Ahora bien, la condicionalidad histórica supera nuestra propia conciencia y determina radicalmente la finitud de toda conciencia hermenéutica —comprensión de sí-, es decir, “autoconocimiento”. La conciencia histórica implica asumir una posición reflexiva en tanto que se considera que todo aquello que es pensado es entregado por la tradición y, como tal, la tarea de la conciencia histórica frente a la tradición es la interpretación de aquello que viene del pasado, que es reflexionado en el presente y que se proyecta en el futuro: la historicidad constituye el espacio y el horizonte de la interpretación.

La comprensión como fenómeno hermenéutico devuelve la universalidad — que de suyo la compone- a la constitución óntica de lo comprendido en cuanto determina lingüísticamente su sentido universal como interpretación, pues "la lingüisticidad de nuestra experiencia del mundo precede a todo cuanto puede ser reconocido e interpretado como ente” (2004: 539). El logos opera la estructuración interna del ser en tanto que lenguaje y las referencias sobre el mundo: el lenguaje es la medida de todos los mundos humanos. El empalme entre la comprensión obtenida desde la tradición como elemento comprensivo y comprensor y la experiencia vital como seres humanos racionales y sociales, da la posibilidad no sólo de comprender nuestro mundo, sino que de transformarlo, cuestionarlo, criticarlo y reorientarlo como "gestores de interpretación” y "herederos de sentido".

El problema hermenéutico de comprensión —en tanto participación y apertura y no manipulación y control; en tanto experiencia y no conocimiento; en tanto dialéctica y no metodología- estribaría, entonces en ¿Cómo se establece la conexión entre el horizonte del presente y el horizonte del pasado para que pueda realizarse la comprensión? Es necesario que uno y otro horizonte se "mezclen" o se "fundan", produciendo una tensión radical entre lo transmitido por la tradición y la situación hermenéutica abierta por la pregunta, por la interpelación de la historia. La historicidad enlaza el 
La apropiación de(l) sentido: Las experiencias hermenéuticas

horizonte presente, que se mueve con el sujeto, y con el horizonte pasado ahora unido por la tradición al presente.

Comprender es un proceso de fusión de presuntos "horizontes para sí mismos": pasado y presente fundidos en "tradición horizóntica" o eslabonados en un "horizonte tradicional" en y desde la alteridad históricamente mediada. La fusión horizóntica posibilitada por el lenguaje revela el acontecimiento significante de la "unidad del sentido" desde un diálogo histórico transmitido y establecido por la tradición: el diálogo es la epifanía lingüística como totalidad de significatividad de(l) sentido.

El lenguaje proporciona la posibilidad de que exista un mundo y de que se manifieste al hombre como mundo. Es decir, no como una totalidad ordenada de cosas, sino como una totalidad ordenada de significaciones, pues el "mundo" y lo real comparecen como sentido, como "efecto" de la mediación lingüística: todo lenguaje comporta una interpretación del mundo y expresa la finitud del ser.

Tal como se ha venido planteando aquí, el lenguaje es una suerte de "mundo intermedio universal" que sirve de eje para entender la experiencia hermenéutica en vistas del horizonte histórico consensuado dialógicamente. Y como medio universal en el que se realiza la comprensión misma; y la forma de realización de la comprensión es la interpretación. El lenguaje de la razón es el lenguaje que puede ser comprendido; la interpretación no es un medio para alcanzar la comprensión sino que aquélla se introduce, por sí misma, en el contenido de lo que se comprende.

En fin, la historia interpretada lingüísticamente y compartida dialógicamente entre sujetos interpretadores/comprensores o el diálogo solidario que surge de la experiencia vivida precientífica y despojada de métodos reglados, de juicios de valor y opiniones previas, descubre un topos que invoca, exige, expulsa y exhorta hacia el límite que marca la inalcanzable pretensión de sentido; un lugar de inasequible acceso para las múltiples facetas, caras y metamorfosis, orientadas por caminos, atajos y escondrijos: el de una coimplicadora preocupación: la acción de interpretar la "experiencia más descorazonadora que la humanidad ha hecho en este siglo [...:] ver que la razón misma es vulnerable” (2004: 48).

Universidad Católica del Maule Facultad de Ciencias Religiosas y Filosóficas Avda. San Miguel 3605, Talca (Chile) fvergara@ucm.cl 
Fernando J. Vergara

\section{BIBLIOGRAFÍA}

GADAMER, Hans-Georg. Verdad y método I. Salamanca: Sígueme, 2005. - Verdad y método II. Salamanca: Sígueme, 2004.

------- El problema de la conciencia histórica. Madrid: Tecnos, 1993.

DOMINGO MORATALLA, Agustín. El arte de poder no tener razón: la hermenéutica dialógica de Gadamer. Salamanca: Universidad Pontificia de Salamanca, 1991.

GARAGALZA, Luis. Introducción a la hermenéutica contemporánea: simbolismo, cultura y sociedad. Barcelona, Anthropos: 2002.

GRONDIN, Jean. Introducción a Gadamer. Barcelona: Herder, 2003.

HEIDEGGER, Martin. Ser y tiempo. Santiago de Chile: Universitaria, 1998.

ORTIZ-OSÉS, Andrés y LANCEROS, Patxi dirs. Diccionario de la existencia. Asuntos relevantes de la vida humana. Barcelona-México: Anthropos-CRIM/UNAM, 2006;

ORTIZ-OSÉS, Andrés y LANCEROS. Patxi dirs. Claves de hermenéutica. Para la cultura, la filosofía y la sociedad. Bilbao: Universidad de Deusto, 2005.

ORTIZ-OSÉS, Andrés y LANCEROS, Patxi dirs. Diccionario de hermenéutica. Una obra interdisciplinar para las ciencias humanas. Bilbao: Universidad de Deusto, 2004.

RICOEUR, Paul. Del texto a la acción. Ensayos de hermenéutica II. Buenos Aires: F.C.E., 2001.

VATTIMO, Gianni. Ética de la interpretación. Barcelona: Paidós, 1991.

VATTIMO, Gianni. El fin de la modernidad. Nihilismo y hermenéutica en la cultura posmoderna. Barcelona: Gedisa, 1996. 